\title{
Increased Poliovirus-Specific Intestinal Antibody Response Coincides with Promotion of Bifidobacterium longum-infantis and Bifidobacterium breve in Infants: A Randomized, Double-Blind, Placebo-Controlled Trial
}

\author{
CATHERINE MULLIÉ, ASMAE YAZOURH, HÉLÈNE THIBAULT, MARIE-FRANÇOISE ODOU, \\ ELISABETH SINGER, NICOLAS KALACH, ODILE KREMP, AND MARIE-BÉNÉDICTE ROMOND \\ Unité d'étude de la translocation bactérienne [C.M.], Faculté de Pharmacie d'Amiens, Université de \\ Picardie, 80037 Amiens Cedex 1, France; Laboratoire de Bactériologie-Virologie [A.Y., M.-F.O., E.S., \\ M.-B.R.], Faculté des Sciences Pharmaceutiques et Biologiques, Université de Lille 2, 59006 Lille Cedex, \\ France; Conservatoire National des Arts et Métiers [H.T.], 75015 Paris, France; and Hôpital Pédiatrique \\ St Antoine [O.K.], Université Catholique de Lille, 59000 Lille, France
}

\section{ABSTRACT}

\begin{abstract}
To determine whether the size of the intestinal bifidobacteria population can influence the immune response to poliovirus vaccination in infants, we set up a randomized, placebocontrolled trial. From birth to $4 \mathrm{mo}$, infants were given a fermented infant formula (FIF) or a standard formula (placebo). Bifidobacteria were quantified monthly in infant stools. Antipoliovirus IgA response to Pentacoq ${ }^{\circledR}$ was assessed before and 1 mo after the second vaccine injection. Thirty infants were randomized, and 20 completed the study (nine in the placebo group and 11 in the FIF group). Fecal bifidobacterial level was significantly higher with the FIF group at 4 mo of age $(p=0.0498)$. Furthermore, B. longum/B. infantis carriage was higher at 4 mo in the FIF group $(p=0.0399)$. Antipoliovirus IgA titers increased after Pentacoq ${ }^{\circledR}$ challenge $(p<0.001)$, and the rise was significantly higher in the FIF group $(p<0.02)$. Antibody titers
\end{abstract}

correlated with bifidobacteria, especially with $B$. longum $/ B$. infantis and $B$. breve levels $(p<0.002)$. Infants who harbored $B$. longum/B. infantis also exhibited higher levels of antipoliovirus IgAs $(p<0.002)$. In conclusion, the present results indicate that antipoliovirus response can be triggered with a fermented formula that is able to favor intestinal bifidobacteria. Whether this effect on the immune system is achieved through the bifidogenic effect of the formula (mainly through B. longum/B. infantis and $B$. breve stimulation) or directly linked to compounds (i.e. peptides) produced by milk fermentation remains to be investigated. (Pediatr Res 56: 791-795, 2004)
Abbreviations
FIF, fermented infant formula
IPV, inactivated poliovirus vaccine

Breast milk is known to protect newborns from infection. As secretory $\operatorname{IgA}$ is the predominant immunoglobulin in breast milk (1), it supposedly acts locally in the infant gut as a first line of defense against foreign antigens. Secretory $\operatorname{IgA}$ is well adapted to persist in the gastrointestinal tract because it is resistant to digestive enzymes (1) as shown by its detection in feces (2). It thus can bind to gastrointestinal pathogens and interfere with their attachment to mucosal cells (2-4).

Received July 16, 2003; accepted May 7, 2004.

Correspondence: Marie-Bénédicte Romond, Ph.D., Laboratoire de BactériologieVirologie, Faculté des Sciences Pharmaceutiques et Biologiques, Université de Lille 2 , Rue du Pr Laguesse 59000 Lille, France; e-mail: mromond@pharma.univ-lille2.fr Financial support was provided by Blédina SA.

DOI: 10.1203/01.PDR.0000141955.47550.A0
Paradoxically, breast-fed infants have been found to produce their own intestinal secretory $\operatorname{IgA}$ sooner than formula-fed infants (4). This stimulation of the immune system might be related to bacteria that highly colonize the intestinal tract of breast-fed infants, such as bifidobacteria (5-7). Indeed, experimental gut colonization of germ-free mice with human bifidobacteria have already shown an enhanced immune response to rotavirus by increasing anti-rotavirus $\operatorname{IgA}$ production (8). Therefore, bifidobacteria are possibly involved in the priming of the infant immune system.

To establish whether high colonization with bifidobacteria can trigger intestinal immune response, we attempted to enhance the bifidobacterial flora of healthy bottle-fed newborns. To this purpose, we used an infant formula that contains new bifidogenic factors generated through a fermentation process 
implying Bifidobacterium breve strain C50 and Streptococcus thermophilus. The fermented formula did not contain viable bacteria but bifidogenic factors previously shown to promote intestinal bifidobacteria in human flora-associated mice as well as in adults $(9,10)$. In this clinical trial, the possible immunomodulative properties of intestinal bifidobacteria were investigated by quantifying fecal bifidobacterial levels and fecal $\operatorname{Ig} \mathrm{A}$ titers (as markers of mucosal immune stimulation) before and after vaccination with the inactivated poliovirus vaccine (IPV). IPV is known to protect people from illness by inducing circulating neutralizing antibodies, but it elicits poor mucosal IgA response in the intestine (11). An increase in antipoliovirus mucosal $\operatorname{IgA}$ would then indicate specific stimulation of the intestinal immune system.

\section{METHODS}

Case definition. Inclusion criteria were 1) vaginal delivery, 2) gestational age between 38 and $42 \mathrm{wk}, 3$ ) birth weight $>2500 \mathrm{~g}, 4$ ) bottle feeding previously decided by parents, 5) follow-up by one of the study pediatricians, and 6 ) written informed consent of the two parents or the legal guardian. Exclusion criteria were 1) caesarean delivery, 2) twins or multiple births, 3) breast-feeding started before inclusion, 4) associated disease requiring antibiotic administration or likely to interfere with the course of the study, and 5) prescription of a specific diet.

Data set. Infants $(n=34)$ who were born between August 1999 and January 2000 at the maternité Pavillon de la Sainte Famille (Clinique du Bois, Lille, France) were assessed for enrollment within the first days after birth. Of them, 30 (seven boys and 23 girls) met inclusion criteria and entered the study (Fig. 1).

Study design. This study was a randomized (block randomization), double-blind, placebo-controlled trial. The study protocol and consent procedures were approved by the local ethics committee (Comité Consultatif des Personnes se prêtant à la Recherche Biomédicale de Lille). The infant formulas were supplied as powder in numbered containers (Blédina SA, Steenvoorde, France). They had the same basal nutrient composition ( $1.45 \mathrm{~g}$ of protein, $8.3 \mathrm{~g}$ of carbohydrates, and $3.5 \mathrm{~g}$ of fat per $100 \mathrm{~mL}$ ). Newborns received the placebo or the fermented infant formula (FIF) from inclusion until 4 mo of age. Respecting the French vaccination program, they received an injection of Pentacoq ${ }^{\circledR}$ (vaccine against diphtheria and tetanus toxoids, poliomyelitis virus, Haemophilus influenzae, and Bordetella pertussis; Pasteur Mérieux Serums and Vaccines, Lyon, France) at 2, 3, and 4 mo. Infants were followed up to the age of 5 mo. Experienced pediatricians carefully collected the history of any gastrointestinal and respiratory infections during monthly visits, as well as filled in a form with children's clinical history, anthropometric data, tolerance, acceptability, and volumes of milk taken at each monthly visit. The pediatricians who monitored tolerance during the study and the people who handled the samples were not aware of which milk the infants were receiving.

Fecal IgA titer determination. Stools were collected for IgA quantification at 3 (before the second Pentacoq ${ }^{\circledR}$ vaccine in-

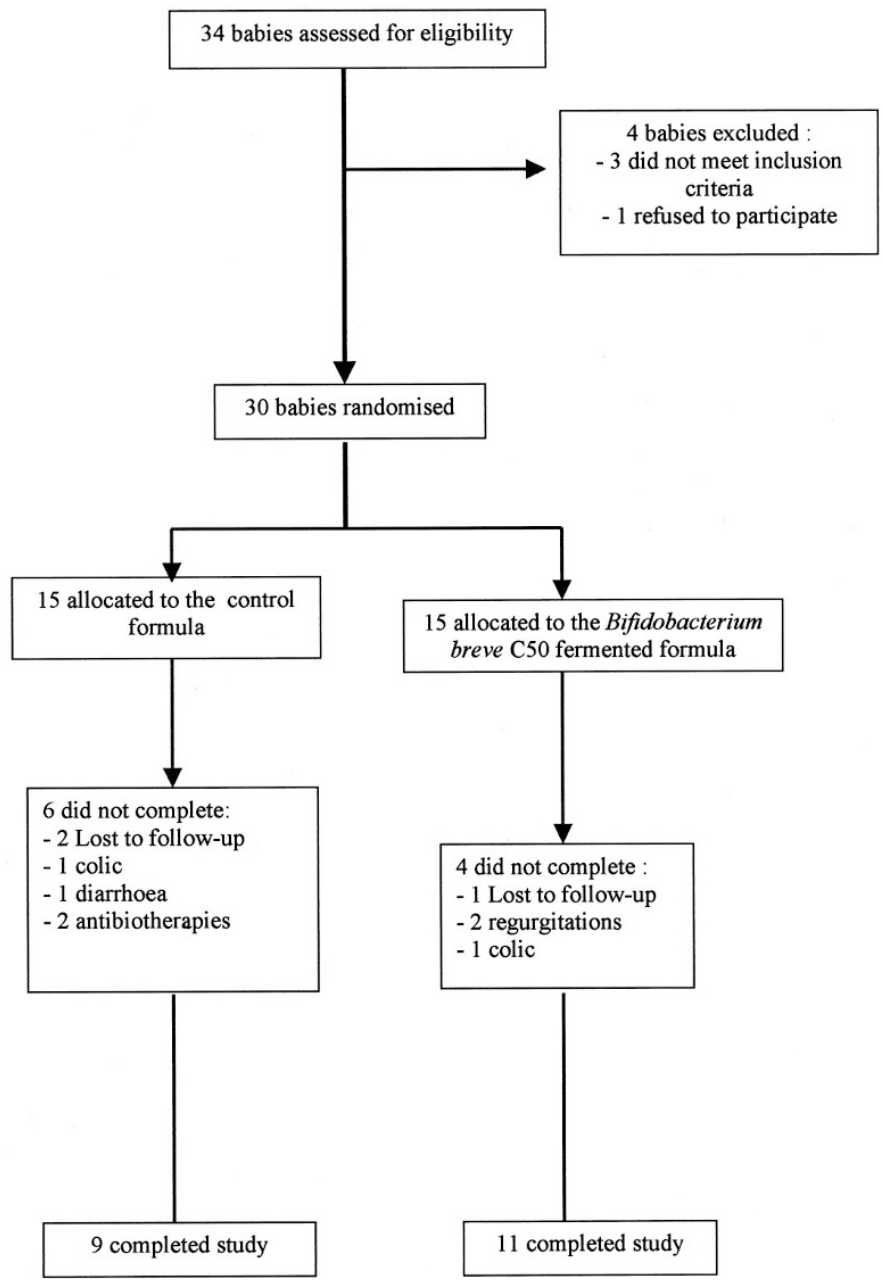

Figure 1. Trial profile.

jection), 3.5, and 4 mo (after the second Pentacoq ${ }^{\circledR}$ vaccine injection). They were immediately frozen until further analysis. Dry stool weight was determined after freeze-drying, and protein content was quantified using the Lowry method (12). Total IgA and anti-poliomyelitis-specific IgA titers were measured by ELISA. Briefly, dry stools were suspended (wt/10 vol) in $0.1 \mathrm{M}$ of PBS buffer (pH 7.4) with protease inhibitors (Sigma Chemical Co., Saint Quentino-Fallavier, France) pepstatin and leupeptin $(1 \mathrm{mg} / \mathrm{mL}), 4$-(2-aminoethyl) benzenesulfonyl fluoride $(50 \mu \mathrm{g} / \mathrm{mL})$, and dry milk powder $(0.05 \mathrm{~g} / \mathrm{mL})$. For the total fecal IgA detection, microplates were coated with goat anti-human secretory component (IgA) antibody (Sigma Chemical Co.) 1:100 (vol/vol) diluted in coating buffer [20 mM $\mathrm{NaHCO}_{3}$ (pH 9.5)]. For antipoliovirus antibody detection, microplates were coated with 1:100 (vol/vol) diluted Sabin vaccine (Copper, Lille, France). After incubation overnight at $4^{\circ} \mathrm{C}$, the wells were washed three times. They were then saturated with $0.1 \mathrm{M}$ of PBS buffer $(\mathrm{pH}$ 7.4) that contained $1 \%$ (wt/vol) BSA and $0.025 \%$ (vol/vol) Tween-20. After three washings, the samples were added to the wells and incubation $\left(1 \mathrm{~h}, 37^{\circ} \mathrm{C}\right)$ was carried out in duplicate for each sample. On each microplate, a negative control [0.1 M of PBS buffer $(\mathrm{pH}$ 7.4)] was included as well as a positive control (IgA from human colostrum). Detection of total or antipoliovirus fecal 
IgA was carried out using horseradish peroxidase-labeled sheep anti-human IgA ( $\alpha$-chain; ICN, Coger, France). The reading was taken at a wavelength of $492 \mathrm{~nm}$.

Rectal flora analysis. Fecal samples to enumerate cultivable bifidobacteria and total cultivable fecal flora were collected at $1,2,3$, and 4 mo, on swabs that allow for the survival of anaerobic bacteria. Appropriate dilutions of the samples were plated onto 1) horse blood agar (Columbia agar base; Oxoid, Dardilly, France) supplemented with glucose $(0.5 \%)$ and cysteine $\cdot \mathrm{HCl}(0.03 \%)$ to enumerate eubacteria, cocci, and clostridia; 2) Beerens and MRS agar for bifidobacteria and lactobacilli; and 3) EMB for enterobacteria. Each type of colony was subcultured on Rosenow broth, Gram-stained, and tested for aerobic susceptibility and catalase production. The sum of the various bacteria recovered gave the total cultivable flora expressed as colony forming units $(\mathrm{CFU}) / \mathrm{mL}$. The counts in cultivable bifidobacteria were expressed as the percentage of the total cultivable flora. Bacteria are considered to belong to the dominant flora when their proportion is $>1 \%$ of the total cultivable flora (13). The detection limit of the method was $0.01 \%$. Bifidobacteria were identified at the species level by a multiplex PCR technique using species-specific primers previously described (14).

Statistical analysis. IgA titers and total cultivable bifidobacterial proportions were analyzed by nonparametric ANOVA for repeated measures (Conover's method). Fisher exact test was used to evaluate the difference in colonization percentages by the various bifidobacterial species between the feeding groups. Mann-Whitney test was used to compare antipoliovirus IgA titers between infants who harbored B. longum-infantis at 4 mo and those who did not. Spearman rank correlation coefficient was used to correlate fecal bacterial counts with $\operatorname{Ig}$ A concentration. Bacterial proportions are reported as mean \pm $\mathrm{SD}$, and $\operatorname{IgA}$ titers are expressed as the median.

\section{RESULTS}

Infants. Twenty of 30 infants completed the study (Fig. 1). Causes of premature ending were similar in the two groups: infants lost to follow-up (two placebos and one FIF) did not complete the study protocol (the 4-mo visit was usually missed). Regurgitations, diarrhea, and colics were alleviated by a change in the infant formula administered (two placebos and three FIF). Two placebo infants experienced rhinopharyngitis followed by bronchitis. Their medical treatment included antibiotic administration (amoxicillin/clavulanic acid association for one of them and josamycin for the other), leading to their exclusion from the study. No link between the formula administered and the occurrence of health problems could be established. All of the infants recovered without further troubles. The two final groups (nine placebos and 11 FIF) were comparable for sex ratio, weight, length, head circumference at birth, age at each monthly visit, clinical tolerance, formula intake, and growth.

Fecal IgA titers. Total IgA titers were similar in both groups and did not increase after vaccination (data not shown). Antipoliovirus IgA titers were similar at 3 and 3.5 mo (median: 160 and $200 \mathrm{U} / \mathrm{g}$ of dry stools and 80 and $160 \mathrm{U} / \mathrm{g}$ of dry stools in the placebo and the FIF groups, respectively; Fig. 2). At 4 mo, antipoliovirus $\operatorname{IgA}$ titers were significantly increased in both groups after vaccination (median: $1280 \mathrm{U} / \mathrm{g}$ of dry stools and $507 \mathrm{U} / \mathrm{g}$ of dry stools for the FIF and placebo groups, respectively; $p<0.001$, nonparametric ANOVA for repeated measures). Moreover, the increase in antipoliovirus IgA titers was greater in the FIF group $(p<0.02$, nonparametric ANOVA for repeated measures; Fig. 2).

Intestinal colonization with bifidobacteria. Bifidobacteria were detected in all infants. Infants who received the FIF had a higher mean proportion of cultivable bifidobacteria $(p=$ 0.0498, nonparametric ANOVA for repeated measures; Fig. 3). Species identification was carried out successfully for all but one strain, subsequently referred to as Bifidobacterium sp. (Table 1). Whatever the feeding, the most frequently isolated species were $B$. bifidum, $B$. breve, and $B$. longum-infantis. Carriage of $B$. longum-infantis was shown to be susceptible to the type of feeding. In the placebo group, it decreased, whereas it rose over time in the FIF group. In addition, a higher number

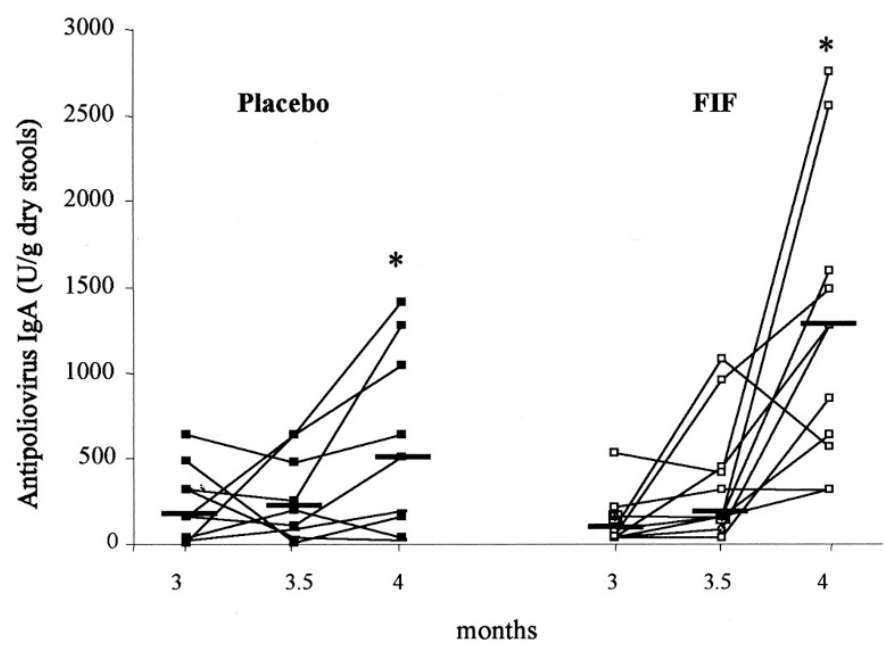

Figure 2. Mucosal antipoliovirus $\operatorname{IgA}$ response to vaccination in placebo ( $n$ $=9)$ and $\operatorname{FIF}(n=11)$ infants. The solid black bar represents the median value in each group. *Significant rise in antipoliovirus IgA at 4 mo in placebo and FIF infants ( $p<0.001$, nonparametric ANOVA for repeated measures).

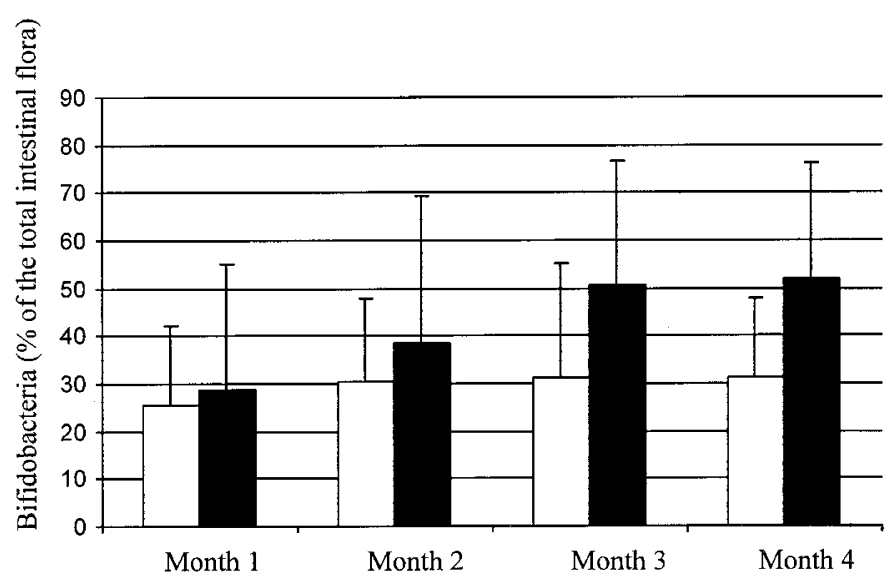

Figure 3. Proportion of cultivable bifidobacteria within the total cultivable flora. Results are expressed as mean with SD. $\square$, placebo $(n=9)$; $\boldsymbol{\square}, \operatorname{FIF}(n$ $=11)$. * Percentage of bifidobacteria significantly higher in FIF infants $(p=$ 0.0498, nonparametric ANOVA for repeated measures). 
of FIF-fed infants were colonized with B. infantis-longum at 4 mo of age ( $p=0.0399$, Fisher exact test; Table 1). Also at 4 mo, FIF infants harbored significantly fewer bifidobacteria that belong to a group of species that are more frequently found in the adult intestinal flora: $B$. angulatum, $B$. adolescentis, $B$. catenulatum, B. dentium, B. pseudocatenulatum, and Bifidobacterium sp. ( $p=0.038$, Fisher exact test).

A positive correlation between total bifidobacteria and antipoliovirus IgA titers was observed on the whole population $(r$ $=0.46, p<0.05$, Spearman test; Fig. $4 A)$. The B. breve/B. longum-infantis subgroup was the sole subgroup within the bifidobacterial species that correlated with antipoliovirus $\operatorname{IgA}$ titers $(r=0.73, p<0.002$, Spearman test; Fig. $4 B)$. In any case, whichever group to which they belonged, infants who harbored detectable levels of $B$. longum-infantis showed higher antipoliovirus IgA titers at 4 mo than those without $(p<0.002$, Mann-Whitney test).

\section{DISCUSSION}

Breast-feeding was previously shown to stimulate response to vaccination. Pickering et al. (15) observed that 2 mo of breast-feeding specifically enhanced antibody responses to oral poliovirus immunization compared with infant formulas. Different mechanisms have been suggested to explain this adjuvant effect (16). One of them might involve the gastrointestinal flora, as breast-feeding predominantly stimulates the growth of $B$. breve and B. longum-infantis (5-7), and as gastrointestinal flora is described as a major stimulator of the gut associated lymphoid system (17-21).

In the present study, FIF induced a rise in the proportion of cultivable intestinal bifidobacteria and specifically favored the carriage of B. longum-infantis. At the same time, a greater rise in IgAs was observed in the FIF group, and the response to poliovirus vaccination was related to the bifidobacterial proportion in the total flora. The species B. longum-infantis and $B$. breve were strongly suspected of supporting the priming of the antipoliovirus response as their proportion in the flora correlated with antibody titers. More specific, B. longum-infantis carriage was linked with higher IgA titers in the infant stools, whatever the feeding group. Contrasting, the most common bifidobacterial species found in infants, B. bifidum, was not associated with higher IgA titers. Thus, only a subgroup of the bifidobacterial genus that colonized the infant gut correlated with antipoliovirus IgA response. These results suggest that the immune effect is linked to a specific bifidobacterial population. However, it is also conceivable that some FIF compounds might directly participate in the elicitation of the immune response. Indeed, the oral feeding of peptides derived from milk fermentation with Lactobacillus helveticus has been reported to increase the number of IgA-producing cells in the mouse small intestine (22). Hence, Bifidobacterium breve C50 might also produce peptides such as these immunomodulative ones during the milk fermentation process.

The analysis of IgA synthesis after vaccination could provide us with some clues as to the mechanism(s) involved in the antipoliovirus antibody elicitation observed in the present survey. Humans and monkeys are the only natural hosts for poliovirus. It is commonly accepted that recognition of CD155 (poliovirus receptor) by poliovirus is the first event in the triggering of specific antipoliovirus IgA production. Indeed, transgenic mice that bear the human receptor for poliovirus showed that the expression of CD155 was necessary for a virus-specific mucosal IgA response (23). In fact, some degree of mucosal immunity can be measured in IPV vaccinees (24), indicating that the first steps of mucosal responses are primed by the inactivated poliovirus vaccine, although the intramuscular administration route avoids close contact with the receptors exhibited on the intestinal cell surfaces. Buisman et al. (25) showed that poliovirus administered to transgenic mice by the peritoneal route was transported to the gut lumen by macrophages, hence inducing local IgA production. A similar transport of vaccinal poliovirus strains is likely to occur in infants after Pentacoq ${ }^{\circledR}$ vaccination. In the present study, specific IgA production cannot be related to recognition between CD155 and either bifidobacteria or compounds of FIF. Otherwise, the increase in fecal antipoliovirus IgA titers would occur independent of vaccination. Consequently, the increased antipoliovirus response observed in the FIF group is possibly the result of an adjuvant effect mediated either through compounds contained in FIF or through intestinal B. longum/B. infantis.

To support this claim, reports of an adjuvant effect on Peyer's patches have already been published for bifidobacteria $(26,27)$, and uptake of bifidobacteria by the digestive tract has previously been shown in infants (28). Bifidobacteria could subsequently be processed and presented to lymphocytes in Peyer's patches (29), and positive cooperation with IgA-producing cells in the gut could result in higher antipoliovirus IgA titers (30).

\section{CONCLUSION}

In conclusion, the feeding of infants with FIF gave promising results in eliciting intestinal immunity. Moreover, the present study supports the view that increased poliovirus-specific intesti-

Table 1. Carriage of Bifidobacterium species throughout the study (placebo, $\mathrm{n}=9 ; \mathrm{FIF}, \mathrm{n}=11$ )

\begin{tabular}{|c|c|c|c|c|c|c|c|c|}
\hline & \multicolumn{2}{|c|}{ Month 1} & \multicolumn{2}{|c|}{ Month 2} & \multicolumn{2}{|c|}{ Month 3} & \multicolumn{2}{|c|}{ Month 4} \\
\hline & Placebo & FIF & Placebo & FIF & Placebo & FIF & Placebo & FIF \\
\hline B. bifidum & $7(78)^{*}$ & $4(36)$ & $7(78)$ & $7(64)$ & $8(89)$ & $10(91)$ & $7(78)$ & $8(73)$ \\
\hline B. breve & $5(55)$ & $7(64)$ & $4(44)$ & $7(64)$ & $2(22)$ & $5(45)$ & $3(33)$ & $4(36)$ \\
\hline B. infantis-longum & $6(67)$ & $5(45)$ & $7(78)$ & $6(54)$ & $4(44)$ & $7(64)$ & $3(33)$ & $9(82) \ddagger$ \\
\hline Others $\uparrow$ & $2(22)$ & $2(18)$ & $4(44)$ & $1(9)$ & $3(33)$ & $2(18)$ & $5(55)$ & $1(9) \S$ \\
\hline
\end{tabular}

* Number of infants carrying the mentioned species $(\%)$.

$\uparrow$ Others include strains belonging to B. angulatum, B. adolescentis, B. catenulatum, B. dentium, B. pseudocatenulatum, and Bifidobacterium sp.

$\ddagger$ Significantly different from the placebo group $(p=0.0399$, Fisher exact test).

$\S$ Significantly different from the placebo group $(p=0.038$, Fisher exact test). 
A

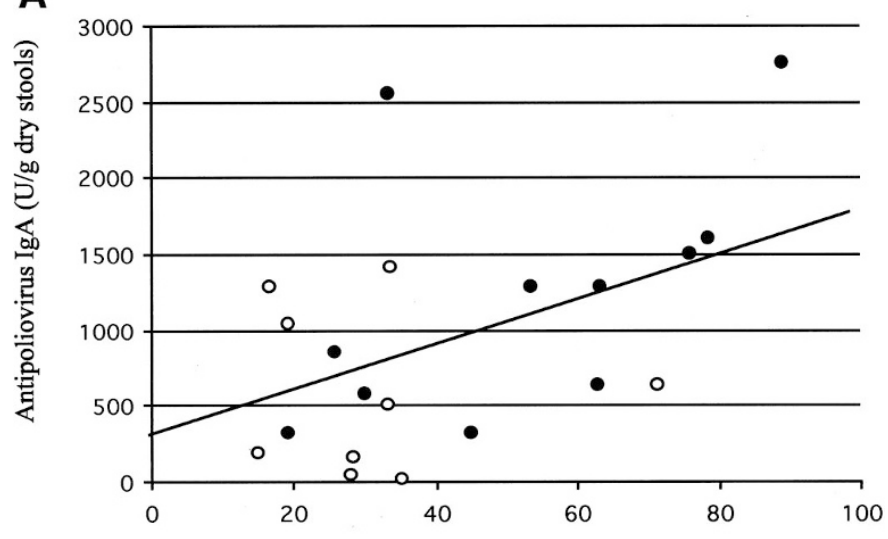

Total cultivable bifidobacteria (\% total cultivable flora)

B

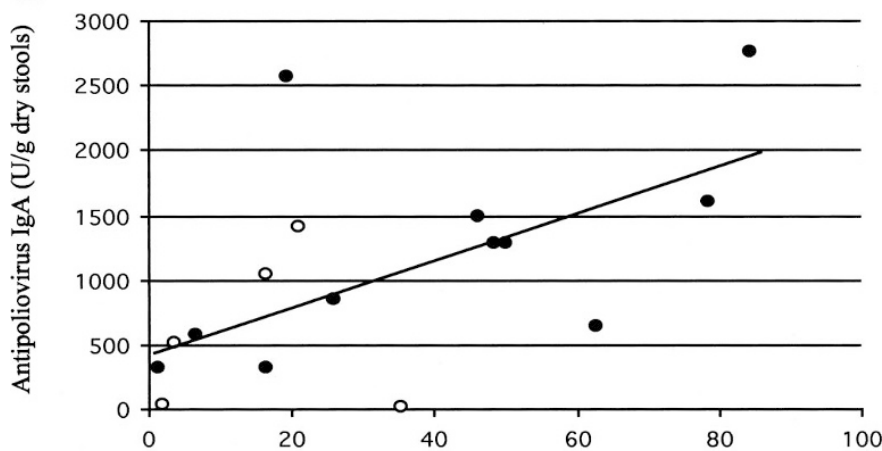

Cultivable B. longum- infantis/B. breve (\% total cultivable flora)

Figure 4. (A) Correlation between the percentage of cultivable bifidobacteria within the total cultivable flora and antipoliovirus IgA titers. $\bigcirc$, infant from the placebo group;, infant from the FIF group. $r=0.46 ; p<0.05$, Spearman test. (B) Correlation between the percentage of cultivable B. longum-infantis and $B$. breve within the total cultivable flora and antipoliovirus IgA titers. $\bigcirc$, infant from the placebo group; $\bullet$ infant from the FIF group. $r=0.73 ; p<0.002$, Spearman test.

nal antibody response coincides in infants with the promotion of $B$. longum-infantis and B. breve but not with B. bifidum. These findings should encourage the performing of a complete analysis of intestinal bifidobacteria at the species level when manipulating the gut flora. Whether a causative link between this promotion and immune system activation can be drawn remains to be elucidated. On the one hand, a positive correlation is clearly observed between antipoliovirus $\operatorname{Ig} \mathrm{A}$ titers and $B$. longum/B. infantis species carriage, whatever the feeding group. On the other hand, however, a possible mechanism involving FIF compounds (i.e. peptides) produced by milk fermentation with a bifidobacterial strain cannot be excluded from immune system activation. Indeed, promotion of some bifidobacterial species might only be a "side effect" of FIF compound activity. Further studies thus are needed to determine the extent of each alternative contribution to specific $\operatorname{IgA}$ stimulation.

Acknowledgments. We thank Dr. Menet and the pediatricians at the Maternité de la Sainte Famille for contribution to newborn inclusions and monthly consultations. We also thank Patrick Devos for providing statistical advice and carrying out part of the statistical analysis, Nathalie Derensy and Fatiha N'Zerwalt for skillful technical assistance, and Cécile Aubert-Jacquin for her help in preparing the manuscript.

\section{REFERENCES}

1. Goldman AS 1993 The immune system of human milk: antimicrobial, antiinflammatory and immunomodulating properties. Pediatr Infect Dis J 12:664-671

2. Davidson LA, Lonnerdal BO 1987 Persistence of human milk proteins in the breast-fed infant. Acta Paediatr Scand 76:733-740

3. Vassilev TL, Veleva KV 1996 Natural polyreactive IgA and IgM autoantibodies in human colostrum. Scand J Immunol 44:535-539

4. Jatsyk GV, Kuvaeva IB, Gribakin SG 1985 Immunological protection of the neonatal gastrointestinal tract: the importance of breast feeding. Acta Paediatr Scand 74:246-249

5. Biavati B, Castagnoli P, Crociani F, Trovatelli LD 1984 Species of the Bifidobacterium in the feces of infants. Microbiologica 7:341-345

6. Benno Y, Sawada K, Mitsuoka T 1984 The intestinal microflora of infants: composition of fecal flora in breast-fed and bottle-fed infants. Microbiol Immunol 28:975-986

7. Harmsen HJ, Wildeboer-Veloo AC, Raangs GC, Wagendorp AA, Klijn N, Bindels JG, Welling GW 2000 Analysis of the intestinal flora development in breast-fed and formula-fed infants by using molecular identification and detection methods. J Pediatr Gastroenterol Nutr 28:19-25

8. Moreau MC 1998 Modulating properties of intestinal bacterial strains, E. coli and Bifidobacterium on two specific immune responses generated by the gut, i.e. oral tolerance to ovalbumin and intestinal IgA anti-rotavirus response in gnotobiotic animals. In: Talwar GP, Nath I (eds) 10th International Congress of Immunology. Bologna, Monduzzi Editore, pp 407-411

9. Romond MB, Ais A, Yazourh A, Romond C 1997 Cell-free wheys from bifidobacteria fermented milks exert a regulatory effect on the intestinal microflora of mice and humans. Anaerobe 3:137-143

10. Romond MB, Ais A, Guillemot F, Bounouader R, Cortot A, Romond C 1998 Cell-free whey from Bifidobacterium breve $\mathrm{C} 50$ fermented milk modifies the colonic microflora in healthy subjects. J Dairy Sci 81:1229-1235

11. Carlsson B, Zaman S, Mellander L, Jalil F, Hanson LA 1985 Secretory and serum immunoglobulin class-specific antibodies to poliovirus after vaccination. J Infect Dis 152:1238-1244

12. Lowry OH, Rosebrough NJ, Farr AL, Randall RJ 1951 Protein measurement with the Folin phenol reagent. J Biol Chem 193:265-275

13. Holdeman LV, Good IJ, Moore WE 1976 Human fecal flora: variation in bacterial composition within individuals and a possible effect of emotional stress. Appl Environ Microbiol 31:359-375

14. Mullié C, Odou MF, Singer E, Romond MB, Izard D 2003 Multiplex PCR using 16S rRNA gene-targeted primers for the identification of bifidobacteria from human origin. FEMS Microbiol Lett 222:129-136

15. Pickering LK, Granoff DM, Erickson JR, Masor ML, Cordle CT, Schaller JP, Winship TR, Paule CL, Hilty MD 1998 Modulation of the immune system by human milk and infant formula containing nucleotides. Pediatrics 101:242-249

16. Hahn-Zoric M, Carlsson B, Jeansson S, Ekre HP, Osterhaus AD, Roberton D, Hanson LA 1993 Anti-idiotypic antibodies to poliovirus antibodies in commercial immunoglobulin preparations, human serum, and milk. Pediatr Res 33:475-480

17. Riordan SM, McIver CJ, Wakefield D, Thomas MC, Duncombe VM, Bolin TD 1999 Serum immunoglobulin and soluble IL-2 receptor levels in small intestinal overgrowth with indigenous gut flora. Dig Dis Sci 44:939-944

18. Ouwehand A, Isolauri E, Salminen S 2002 The role of the intestinal microflora for the development of the immune system in early childhood. Eur J Nutr 41(suppl 1):I32-I37

19. Rhee KJ, Sethupathi P, Driks A, Lanning DK, Knight KL 2004 Role of commensal bacteria in development of gut-associated lymphoid tissues and preimmune antibody repertoire. J Immunol 172:1118-24

20. Vinderola CG, Medici M, Perdigon G 2004 Relationship between interaction sites in the gut, hydrophobicity, mucosal immunomodulating capacities and cell wall protein profiles in indigenous and exogenous bacteria. J Appl Microbiol 96:230-243

21. Schiffrin EJ, Blum S 2002 Interactions between the microbiota and the intestinal mucosa. Eur J Clin Nutr 56:S60-S64

22. LeBlanc JG, Matar C, Valdéz JC, LeBlanc J, Perdigon G 2002 Immunomodulating effects of peptidic fractions issued from milk fermented with Lactobacillus helveticus. J Dairy Sci 85:2733-2742

23. Buisman AM, Sonsma JA, Kimman TG, Koopmans MPG 2000 Mucosal and systemic immunity against poliovirus in mice transgenic for the poliovirus receptor: the poliovirus receptor is necessary for a virus-specific mucosal IgA response. J Infect Dis 181:815-823

24. Herremans TM, Reimerink JH, Buisman AM, Kimman TG, Koopmans MP 1999 Induction of mucosal immunity by inactivated poliovirus vaccine is dependent on previous mucosal contact with live virus. J Immunol 162:5011-5018

25. Buisman AM, Sonsma JA, van Wijk MG, Vermeulen JP, Roholl PJ, Kimman TG 2003 Pathogenesis of poliovirus infection in PVRTg mice: poliovirus replicates in peritoneal macrophages. J Gen Virol 84:2819-2828

26. Yasui H, Ohwaki M 1991 Enhancement of immune response in Peyer's patch cells cultured with Bifidobacterium breve. J Dairy Sci 74:1187-1195

27. Yasui H, Nagaoka N, Hayawaka K 1994 Augmentation of anti-influenza virus hemagglutinin antibody production by Peyer's patch cells with Bifidobacterium breve YIT4064. Clin Diagn Lab Immunol 1:244-246

28. Heine W, Mohr C, Wutzke KD, Radke M 1991 Symbiotic interactions between colonic microflora and protein metabolism in infants. Acta Paediatr Scand 80:7-12

29. Park JH, Um JI, Lee BJ, Goh JS, Park SY, Kim WS, Kim PH 2002 Encapsulated Bifidobacterium bifidum potentiates intestinal IgA production. Cell Immunol 219:22-27

30. Hexham JM, Carayannopoulos L, Capra JD 1997 Structure and function in IgA Chem Immunol 65:73-87 